\title{
Wireless Power Transfer Module Design using Discontinuous Direct Electromagnetic Field
}

\author{
Md. Rokibul Hassan Billah \\ Undergraduate Student \\ American International \\ University-Bangladesh \\ Banani, Dhaka-1213
}

\author{
Md. Al Amin \\ Undergraduate Student \\ North South University \\ Bashundhara, Dhaka-1229
}

\author{
A.B.M. Khalid Hassan \\ Undergraduate Student \\ American International \\ University-Bangladesh \\ Banani, Dhaka-1213
}

\begin{abstract}
In this paper, a new way of wireless power transmission has been described. By using this method, very simple and low cost general purpose wireless power transfer module is possible to design. All the components which will be required in this design are available in any locality.
\end{abstract}

\section{General Terms}

This method of wireless power transfer module is very simple. This particular way of transferring power wirelessly, has been found by applying many different technics with available local components in some practical experiments. This paper describes mostly on wireless power transmission technique. So, anyone will be able to design their own wireless power transfer module with their available components using this method. That means, there are no bindings about choosing components to design a wireless power transfer module while using this method. Anyone can choose any components which are available from their position.

\section{Keywords}

Direct Electromagnetic Field, Wireless Power Transfer, Low Cost Design.

\section{INTRODUCTION}

Wireless power transfers from transmitting end to receiving end through electromagnetic wave (see Figure 1). That means, to design a wireless power transfer module, it is needed to create an electromagnetic field. The transmitter device will generate this electromagnetic field. And the receiving device will have to be placed inside the electromagnetic field to receive the transmitted power. A transmitter device and a receiver device together makes a complete power transfer module. The amount of total transferred power depends on the distance between transmitter and receiver. This amount will decrease with the increase of distance between transmitter and receiver. Moreover, this amount will be varied with some other parameters. Later it will be described when needed.

\section{TRANSMITTER}

The only duty of a wireless power transmitter is to create an electromagnetic field. That is why an inductor coil is needed. To resonate the electromagnetic wave, a resonance capacitor is needed. And a DC power source or Battery is needed to provide the power.

Now, this algorithm states that, energy can be transferred wirelessly by providing a discontinuous DC power to the transmitter coil. That means, it is not necessary to apply always an alternating power to the transmitter coil. Or more accurately it can be said that, an alternating magnetic field is not mandatory to transfer electricity wirelessly (see Figure 1). Here, the provided current through the transmitter coil will not change direction. It will just be discontinuous, so that a discontinuous direct electromagnetic field can be created (see Figure 1).

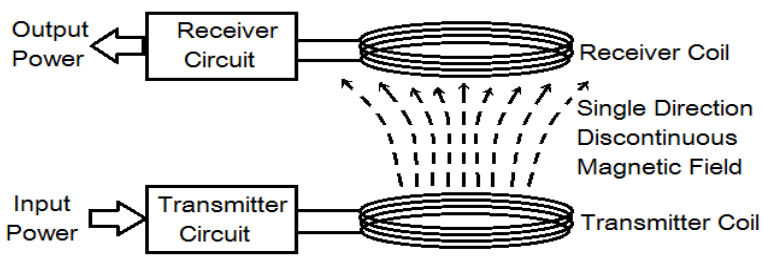

Fig 1. Transferring power wirelessly through single direction discontinuous magnetic field

So a switching device is required to provide a discontinuous power to transmitter coil. Now the wireless power transmitter design will be very simple. The basic wireless power transmitter circuit is given bellow (see Figure 2).

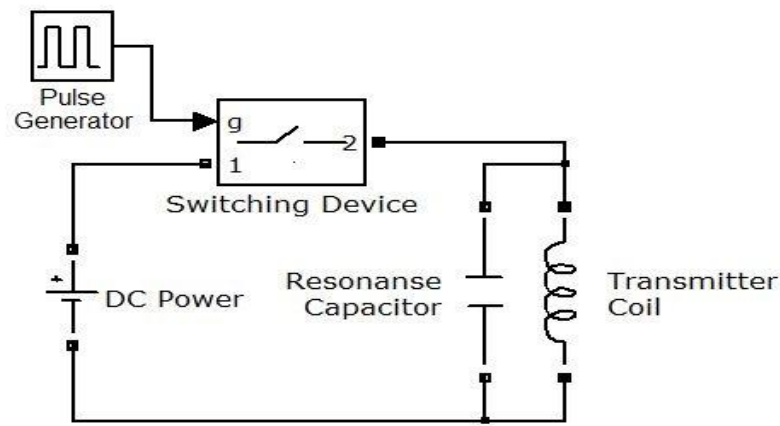

Fig 2. Basic wireless power transmitter circuit

In this circuit, if a 12 volt DC power supply or 12 volt battery is been used. And a 5 volt pulse signal is been applied to the switching device. Then the input signal of the transmitter coil and resonance capacitor will look like the figure given bellow (see Figure 3).

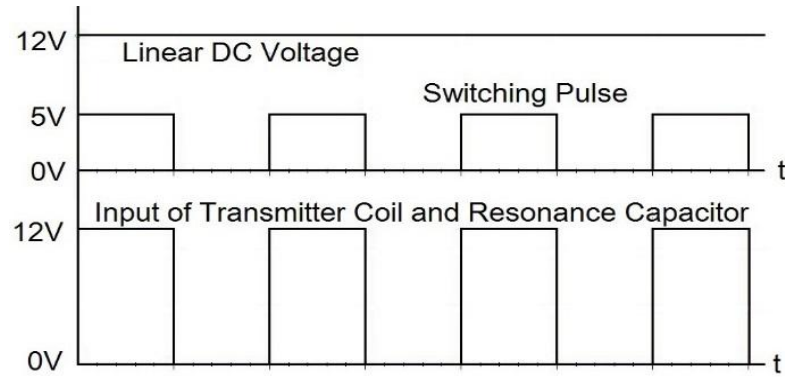

Fig 3. Input signal of transmitter coil and resonance capacitor 
Now, all components of above wireless power transmitter circuit will be described in details bellow.

\subsection{Inductor Coil}

The material of transmitter coil will have to be magnet wire or magnet enameled wire. This magnet wire or magnet enameled wire is a copper or aluminum wire which has an extra thin insulator coating on its outer surface. There are different gauges of magnet wire available, where any gauge can be chosen. But, choosing greater gauge of wire increases the amount of transferred power. For general purpose wireless power transfer module, wire gauge should be 22 or greater. Yet, it depends on application. Now, an inductor coil have to be made with this magnet wire by giving a number of turns. Any number of turns can be given but, the amount of transferred power increases with increase of number of turns in inductor coil. Moreover, any shape and size of inductor coil can be used (see Figure 4). Yet, it also depends on application.

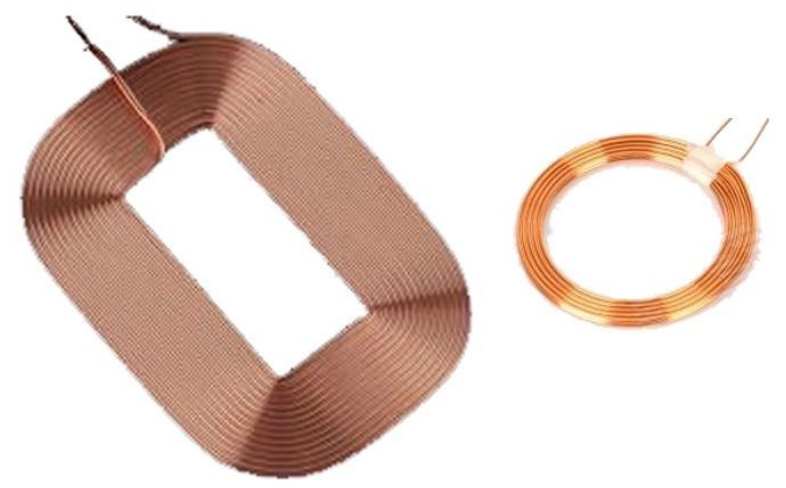

Fig 3. Different sizes and shapes of inductor coil

\subsection{Resonance Capacitor}

A resonance capacitor is needed in parallel with the inductor coil to get the best performance. There are different types of capacitors are available. Any type of capacitor can be selected for this purpose, but Film Capacitor will give the best performance. The value of the capacitor will depend on the inductance of inductor coil. So, at first the inductance of the inductor coil will have to be measured. Then, the required capacitance can be found using the equation given bellow.

We know, at resonance,

$$
X_{L}=X_{C}
$$

Or,

$$
2 \pi f L=\frac{1}{2 \pi f C}
$$

So,

$$
C=\frac{1}{(2 \pi f)^{2} L}
$$

Here,

$$
\begin{aligned}
& C=\text { Required capacitance }(F) . \\
& L=\text { Inductance of coil }(H) . \\
& f=\text { Switching frequency }(\mathrm{Hz}) .
\end{aligned}
$$

The switching frequency will be described later. And it also be set by designer. So, by providing the value of $\mathrm{L}$ and $\mathrm{F}$, the required $\mathrm{C}$ will be found.

\subsection{Power}

In this device, a DC power supply or battery is required. If the available source is AC source, then it has to be converted into dc power. For example, if the wireless power transfer module takes power from the grid, then at first it has to be stepped down by using a step down transformer and then it has to be converted into DC by using a full wave rectifier. After that, a high value of bipolar capacitor have to be used to get a pure linear DC power. Without a linear DC power, the performance of wireless power transfer module will decrease.

\subsection{Switching Device}

The DC power have to be switched frequently to get a discontinuous DC power. In most of the cases, at the time of electrical switching, the ICs get burned before starting the performance. If any power IC can operate this operation, then also power doesn't transfer wirelessly due to the leakage current. For this reason, in every working cycle, the source voltage is been found in first half cycle but, exact zero voltage is not been found in last half cycle (see Figure 4). The amount of leakage current is very small and negligible for general purpose circuits. But it is not negligible for this circuit. Because, this small amount of current hampers wireless power transmission. So, such a switching IC will have to be chosen which will give an exact zero voltage at last half cycle. Otherwise, a mechanical switching device will have to be used here.
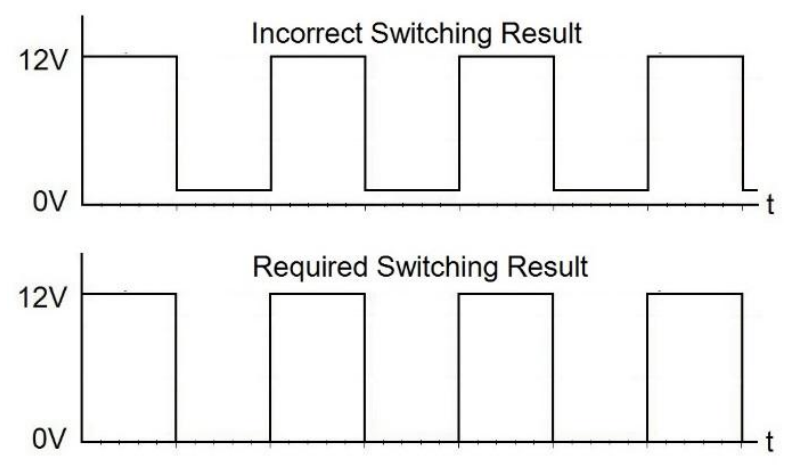

Fig 3. Incorrect Switching Result and Required Switching Result

For example, a high power relay can be used, if it can tolerate. In that case, any multi vibrator IC or any timer IC or any microcontroller IC can be used to generate the switching pulses. Or, the switching device can be made using a servo motor or any other available components. Where, that particular component can be operate using any microcontroller IC or any driver IC.

Now, in terms of switching frequency, the tolerable switching frequency for the used switching device will have to be chosen. But, the amount of transferred energy will increase with the increase of switching frequency.

After connecting the circuit as shown above (see Figure 2), the wireless power transmitter device is complete.

\section{RECEIVER}

Designing a wireless power receiver device is very easy and simple. Here another inductor coil is required to receive the transmitted wireless power. The way of preparing this inductor coil is as same as transmitter coil. It is not mandatory to prepare exactly same size coil for both transmitter and receiver. But, same size inductor coil in both cases will give the best result. Then a resonance capacitor will have to be 
connected in parallel with receiving coil in the same way. Now, the power will be received. And, the received power will be AC power. That means, a full wave rectifier will have to be used to get DC power. Then, a greater value of capacitor will have to be used to get a linear DC voltage. As, the amount of received power varies with the distance between transmitter coil and receiver coil, a voltage regulator can be used to get a fixed voltage in a several range. In that case, the voltage regulator value should have to be lower than input. And, the range of fixed value output will increase with the increase of difference between input voltage and regulated voltage. The basic circuit of wireless power receiver device is given below (see Figure 4).

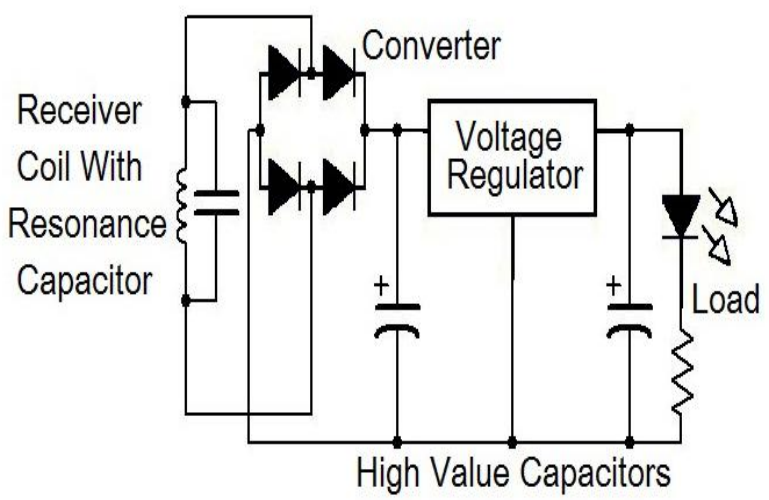

Fig 4. Basic wireless power receiver circuit

Now, by connecting all components in the way which has been shown in above (see Figure 4), the receiver device of wireless power transmission module will be completed.

\section{RESULT}

A wireless power transmitter and a wireless power receiver together makes a complete wireless power transfer module. Several wireless power transfer module has been designed by us with different available components using this method and all of them worked correctly. Comparing with existing general purpose wireless power transfer modules, all the modules which have been built with this method is very simple as well as low cost.

\section{CONCLUSION}

Like existing wireless power transfer modules, here more than one receiver can be used with a single transmitter. But, in that case, like others, the same amount of power will be divided into all receivers.

\section{ACKNOWLEDGMENT}

We are expressing deep respect and giving special thanks to Mr. Ebad Zahir, Special Assistant (Department of EEE), Assistant professor (Faculty of Engineering), American International University-Bangladesh, for all of his inspirations and motivational words in different aspects of study life.

\section{REFERENCES}

[1] Alanson P. Sample, David T. Meyer, and Joshua R. Smith, 2010. Analysis, Experimental Results, and Range Adaptation of Magnetically Coupled Resonators for Wireless Power Transfer, IEEE Xplore.

[2] S. Sheik Mohammed, K. Ramasamy, T. Shanmuganantham, 2010. Wireless Power Transmission - A Next Generation Power Transmission System, International Journal of Computer Applications, Vol. 1, No. 13.

[3] Chang-Jun Ahn, Takeshi Kamio, Hisato Fujisaka, Kazuhisa Haeiwa, 2011. Prototype of $5.8 \mathrm{GHz}$ Wireless Power Transmission System for Electric Vehicle System, 2nd International Conference on Environmental Science and Technology, IPCBEE vol.6.

[4] Johnson I. Agbinya, 2012. Wireless Power Transfer, River Publishers, Singapore.

[5] Wireless Power, Online, 2015. Available: http://www.instructables.com/id/Wireless-PowerTransmission-Over-Short-Distances-U/.

[6] Low-Power Wireless Charging, Online, 2015, Available: http://www.instructables.com/id/Low-Power-WirelessCharging/

[7] Wireless Power, Online, 2015, Available: http://en.wikipedia.org/wiki/Wireless_power. 